\title{
Do Age-related Differences in the Incidence of Mumps Deafness Reflect a True Difference or a Misclassification of Mumps Deafness?
}

\author{
Takashi Fujiwara ${ }^{1}$ and Yohei Maeda ${ }^{2}$ \\ ${ }^{1}$ Department of Public Health Research, Kurashiki Clinical Research Institute, Okayama, Japan \\ ${ }^{2}$ Department of Otorhinolaryngology-Head and Neck Surgery, Osaka University Graduate School of Medicine, Osaka, Japan \\ Received January 7, 2021; accepted January 7, 2021; released online March 20, 2021
}

Copyright $\odot 2021$ Takashi Fujiwara et al. This is an open access article distributed under the terms of Creative Commons Attribution License, which permits unrestricted use, distribution, and reproduction in any medium, provided the original author and source are credited.

\section{To the Editor:}

We read the article by Takagi et al with great interest and appreciate their effort to assess the incidence of mumps deafness in mumps cases. ${ }^{1}$ In a claim-based database study, the authors found that the incidence of mumps deafness was 8.4 times higher in adolescents and adults than in children (73.6 per 10,000 patients for adolescents and adults and 6.6 for children). However, the estimate might be invalid because of the definitions of mumps and mumps deafness used.

The authors used the "B26 code from the International Statistical Classification of Diseases and Related Health Problems, $10^{\text {th }}$ Revision (ICD-10)" to identify mumps cases. The validity of the claim diagnosis code has not been evaluated in Japan, ${ }^{2}$ and we are concerned regarding the underestimation of mumps due to mumps patients seen in a clinical setting who are not given a B26 code. The treatment of mumps is analgesics and cooling the affected area, and there is no specific medication for mumps. When a physician sees a mumps patient, the physician needs to give the patient an analgesia-related ICD-10 code (eg, headache or lower ear pain) for insurance, but does not need to give the ICD-10 B26 code. In Japan, sentinel surveillance is conducted, and there are 0.431 to 1.356 million suspected mumps cases annually. ${ }^{3}$ The Japanese Medical Data Center (JMDC) database is based on employment-based health insurance claims, and it mainly covers the population younger than 65 years. ${ }^{4}$ The database contained 5.5 million subjects as of June $2018,{ }^{1}$ and accounted for about $6.1 \%$ of the total population of Japan younger than 65 years. ${ }^{5}$ Based on a sentinel surveillance rate of $6 \%$, there should be 26,860 to 81,360 cases of pediatric mumps annually. However, Takagi et al identified only 62,551 pediatric mumps cases during their 12-year study.

The authors also used an "acute sensorineural deafness and mumps antibody test in the same month" as the third definition of mumps deafness. Claim-based databases, including the JMDC database, do not have the results of laboratory tests. If a patient has acute sensorineural deafness and has the mumps antibody test to rule out mumps deafness, the patient is classified as "mumps deaf" in the study. Indeed, otolaryngologists occasionally perform the mumps antibody test on acute sensorineural deaf patients without swelling of the parotid glands. Since one of the main cause of pediatric acute sensorineural deafness is mumps deafness, the definition would be acceptable for children. However, mumps deafness is an unusual cause of adult acute sensorineural hearing loss, with the major cause being sudden hearing loss. The incidence of sudden hearing loss increases with age and is about eight times higher in adolescents and adults than in children, ${ }^{6}$ and the age-related difference in sudden hearing loss is similar to that observed in Takagi et al's study.

Therefore, a major limitation of the study by Takagi et al is that the lack of a validation study of the outcome definition might lead to imprecise case definitions for mumps and mumps deafness. Consequently, the age-related difference they observed would not be the true difference in the incidence of mumps deafness.

\section{ACKNOWLEDGMENTS}

We would like to thank textcheck (https://www.textcheck.com/) for English language editing.

This study was not funded by third parties, and the fee for English language editing was paid privately.

Contributions: TF drafted the manuscript, and YM revised the manuscript critically. All authors approved the submitted manuscript.

Conflicts of interest: During the past 3 years, TF receive personal fees from Japan Council for Quality Health Care and Real World Data Co, Ltd.

\section{REFERENCES}

1. Takagi A, Ohfuji S, Nakano T, Kumihashi H, Kano M, Tanaka T. Incidence of mumps deafness in Japan, 2005-2017: analysis of Japanese Insurance Claims Database. J Epidemiol. 2020 Oct 24. doi:10.2188/jea.JE20200233. Epub ahead of print.

2. Koram N, Delgado M, Stark JH, Setoguchi S, de Luise C. Validation studies of claims data in the Asia-Pacific region: a comprehensive review. Pharmacoepidemiol Drug Saf. 2019;28:156-170.

3. National Institute of Infectious Disease. Mumps (infectious parotitis) in Japan, as of July 2013. Infectious Agents Surveillance Report. 2013;34:219-220. https://www.niid.go.jp/niid/en/iasr-vol33-e/865iasr/4222-tpc402.html; Accessed 29.12.2020.

4. Hamada K, Aoki K. Application of Japanese Claims Database to 
pharmacovigilance activity in pharmaceutical industry; Analysis on cancer incidences and usage of anticancer agents. Jpn J Pharmacoepidemiol. 2012;17:145-153 (in Japanese).

5. Statistics Bureau, Ministry of Internal Affairs and Communications. Population Estimates by Age (Five-Year Groups) and Sex. https:// www.stat.go.jp/data/jinsui/pdf/202012.pdf; Accessed 29.12.2020.

6. Fujiwara T, Okada M, Yoshida T, Hakuba N, Hato N, Gyo K. The incidence of sudden sensorineural hearing loss in Ehime prefecture in Japan 2012-2013. Ehime Med J. 2014;33:182-186. 